\title{
GRPR Overexpression Positive
}

National Cancer Institute

\section{Source}

National Cancer Institute. GRPR Overexpression Positive. NCI Thesaurus. Code C158729.

An indication that overexpression of GRPR has been detected in a sample. 